\title{
The Influence of Socio-Cultural Factors in Access to Healthcare in Kenya: A Case of Nairobi County, Kenya
}

\author{
Davies N. Chelogoi, Fred Jonyo, Henry Amadi \\ Department of Political Science and Public Administration, University of Nairobi, Nairobi, Kenya \\ Email: davischelogoi916@gmail.com
}

How to cite this paper: Chelogoi, D. N., Jonyo, F., \& Amadi, H. (2020). The Influence of Socio-Cultural Factors in Access to Healthcare in Kenya: A Case of Nairobi County, Kenya. Open Journal of Social Sciences, 8, 328-347.

https://doi.org/10.4236/jss.2020.85023

Received: September 3, 2019

Accepted: May 23, 2020

Published: May 26, 2020

Copyright ( 2020 by author(s) and Scientific Research Publishing Inc. This work is licensed under the Creative Commons Attribution International License (CC BY 4.0).

http://creativecommons.org/licenses/by/4.0/

\begin{abstract}
Access to public healthcare in Nairobi County is unequal among social classes. Lower social classes have worse healthcare than either the upper or the middle classes. These health inequalities are correlated with socio-economic inequalities. The higher socio-economic classes have better access to healthcare than the lower socio-economic classes. Higher incomes, education, employment and wealth result in better health of the households in the County. Unequal access to healthcare contributes to disparities in health status, increases costs for both the insured and the uninsured. Lack of access to healthcare reduces disposable incomes, particularly burdening the lower income households. These households cannot afford the care they need. This has forced them to forego such care altogether. The objectives of the study were three, namely: to evaluate the influence of demographic variables in access to public healthcare, to evaluate the influence of socio-cultural factors in access to public health care, and to evaluate the influence of institutional factors in access to public healthcare. The study used descriptive design, specifically, cross-sectional design for collection, measurements and analysis of data. The study took place in Nairobi County. The target population was households living in Nairobi County, where the sample was drawn from. The sampling techniques included multi-stage random sampling, random sampling, stratifies random sampling, cluster random sampling, convenient sampling and purposive sampling. The sample size was obtained using Chadha's formula (2006) to arrive at 1066 sample size. Data collection instruments included observations, face-to-face interviews, questionnaires, in-depth interviews and focus group discussions. Qualitative data was analyzed thematically but quantitative data was analyzed using descriptive statistics. Data was analyzed using SPSS version 23. The results show that there were positive correlations between independent and dependent variables. The $P$-value was statistically significant.
\end{abstract}


The results were not due to random chance and that $P-0.01<0.05$ and this confirms a positive relations ships between the variables. The relationships were mutually inclusive and highly correlated. On that basis, the null hypotheses were rejected and the alternate hypotheses accepted. The results show that demographic (disposing), socio-cultural (need) and institutional (enabling) factors influence access to healthcare. Socio-economic factors should be addressed to benefit all the households. Socio-cultural factors should be distributed fairly among the households. Health systems should be improved and adequately financed to provide the requisite resources to all the households.

\section{Keywords}

Social Class, Access to Healthcare, Health Inequalities and Socio-Economic Status

\section{Introduction}

Access to healthcare among the various social groups is unequal in both developed and developing countries. In the United States, access to healthcare is unequal among families with different socio-economic backgrounds. For example, low income families have less access to healthcare, and this explains why they have high infant mortality rates compared to families of high incomes. Evidence also show that African Americans, Red Indians and Hispanics have less access to healthcare due to their racial backgrounds compared to the majority whites (Barr, 2008). Socio-economic and racial factors play a key role in the distribution of access to healthcare.

In France, access to healthcare for manual workers is less compared to professional workers. Professional workers have better socio-economic resources, defined by income, occupation and education, which positively influence access to healthcare. The manual workers have less of these socio-economic resources, hence, the reason for not having good access to healthcare. In this case, occupation or employment statuses have some influence on access to healthcare.

In Canada, low income population has less access to healthcare compare to middle or high income population (Ibid). As a result of this health differences, life expectancy is lower among the lower income households compared with the higher income households (Wilkinson, 1976). Income in this case appears to have an important influence on access to healthcare. Households with high incomes can purchase healthcare services at private or specialized outlets. But those with less income have limited options and this limits their access to healthcare.

In another study, evidence show that Eskimos have less access to healthcare, due to their origin status, compared to the native Australians. Originality status appears to affect access to healthcare. In this study, the native Australians have better advantages over the non-population. The evidence suggests that the 
non-locals are discriminated against and do not access equal resources and opportunities like the locals. Origin, language (Avila \& Bramlett, 2013) and color appear to be important factors in this study.

Like in the previous publications, low income continues to be an important factor. In Britain, low income population has less access to healthcare compared to the higher income populations (Donabedian, 1990). Income includes wages, salaries, rents, pensions and gratuities among others affect access to healthcare. Those with high incomes have capacities to procure healthcare at all costs. Those with less income have less opportunities or resources to access healthcare.

The studies above show that access to healthcare was unequal among social classes. Socio-economic and cultural factors are distributed unequally and the upper social hierarchies have better access to healthcare, compared to the lower social classes. Health inequality persists despite the fact that these are developed countries with advanced health system. These gaps need further researcher and proportionate policy interventions.

In some Sub-Saharan countries, access to healthcare is still common despite numerous research and policy interventions. The World Bank shows that 50\% of African population has access to modern facilities, and more than $40 \%$ do not even access clean water and sanitation. Immunization has not covered the entire population. This has resulted in high levels of maternal and infant mortalities, despite adequate human and material resources at their disposal.

Access to healthcare among poor Tanzanians is poor compared to the wealthy. This is more pronounced in rural areas, where incomes, education, employment is low compared to the urban areas (Schellenberg et al., 2003). In Uganda, access to healthcare is adversely affected because of poor health systems-poor hospitals, lack of equipment, low staff capacities leading to high infant mortality rates (Donabedian, 1990). The low socio-economic class population have limited options compared to the upper and middle class who can afford health insurance cover and use of private facilities (Brawley, 2000).

Like the developed world, selected Sub-Saharan Africa provides limited access to healthcare for its population. Sub-Saharan Africa is endowed with many natural and human resources, and yet access to healthcare is still a major problem. Socio-economic, socio-cultural and institutional disparities continue to influence access to healthcare. Resources have been expended on health systems, but access to healthcare remains a significant challenge. These countries continue with further research and policy interventions to try to provide access to healthcare to the entire population.

Access to healthcare in Kenya is delivered by Government, Non-Governmental organizations and the private sector. The government runs $41 \%$, the non-governmental organizations $15 \%$, and the private business $43 \%$. The Government owns most of the hospitals, health centers and dispensaries. However, clinics and nursing homes are managed by the private sector. Access to healthcare is provided through a network of over 5000 health facilities countrywide. 
These facilities include the national referral hospitals, county referral hospitals, district and sub-districts, health centers and dispensaries. National referral hospitals are at the top, and they provide complex healthcare services. Kenyatta National Hospital and Moi Referral Hospital lead in providing highly complex healthcare services. However, private hospitals like Nairobi Hospital, Aga Khan and many more others are the equivalent and provide highly sophisticated technology and top qualified personnel. County referral hospitals, district and sub-districts provide less but important health services. At the bottom are the health centers and dispensaries that provide other services, especially primary healthcare. The minority of the population access healthcare through the use of traditional specialists (spiritual healers, bone setters, and herbalists).

Access to healthcare is important for all persons in the population. It increases physical, social and mental health of the individual; it improves the quality of life (IOM, 1993). Access to healthcare is important in promoting and maintaining good health; it helps manage and reduce unnecessary diseases. It also prevents early death besides achieving health equity among the population. Access to healthcare is therefore imperative in order to achieve good health and equity for the whole population (IOM, 1993). Access here includes coverage (Durham, et al. 1998), services (Starfield, 2004), and timeliness (Brotherton et al., 2005).

In this thesis, socio-cultural factors (need) which are culture, values, habits, race, ethnicity, language, social cohesion and social resources/capital are discussed to find out their influence on access to healthcare, amongst others; and institutional (enabling) factors that include policies, governance, infrastructure, health facilities, health financing, insurance and health personnel. The goal is to increase access to healthcare and the objectives are to show the influence of these factors on access to healthcare.

This study focuses on access to healthcare in Nairobi County. The results show that access to healthcare is unequal among the households. Only a small number of households' access healthcare but the majority go without adequate access or no access at all. The majority is the lower socio-economic classes, who have less or no opportunities to access healthcare resources compared to the upper and middle classes. These limitations to access have negative effects on the health and potential of the households affected. This is the knowledge gap that the study attempted to fill. A further research is necessary to fill the gap. Policy makers also need to address all these dimensions including, material (socio-economic), cultural (social resources/capital) factors and institutional that affect access to healthcare.

Descriptive design is used to conduct the research. Data collections will use various methods that include sampling techniques such as multistage random sampling, convenient and cluster random sampling. Collections instruments include in-depth interviews and focus group discussion for objective two, and interviews, key informant interviews, and questionnaires for objective one and three. Descriptive statistics are used to analyze and interpret data. Using SPSS, 
version 23 , the results are analyzed and statistical information provided. The objective of the analysis is to find out the relationships between the variables-independent variables and dependent variables. The analysis shows correlation coefficient, Pearson product correlation, Chi-Square and regression of the variables under investigation.

\section{Definitions of Concepts}

\subsection{Social Class-Independent Variable}

Social class refers to a group of people in a society who have the same socio-economic status. The concept refers to a collection of individuals who share similar conditions. The concept has also been used to refer to a group of people who have similar levels of wealth, influence, and status. Sociologists have used three methods to determine social class: upper, middle and lower class. Gullup has used "five levels to define social class: upper, upper middle, middle, working and lower class". Gilbert defines social class, "as groups of people/families who are more or equal in rank and differentiated from other families above or below them with regard to characteristics such as occupation, income, wealth and prestige".

Social class is therefore a set of concepts used in social sciences and political theory to mean social stratification in which people are grouped hierarchically in social categories, most common being the ones referred presently.

Pierre Bourdien has attempted to explain "social reproduction, the tendency for social class status to be passed down from one generation to the next". This happens because each generation acquires cultural capital (tastes, habits, expectations, skills ...). Ogburn and Nimkoff define social class as "one or two broad groups of individuals who are ranked by members of the community in socially superior and inferior positions".

However, Max Weber defines social class as "class or aggregates of individuals who have the same opportunity of acquiring good, the same exhibited standard of living". Sociologists here see social class as a powerful form of stratification but other layers can be drawn on such factors as age, gender, and ethnicity and so on. In that regard, "placing people within such layers or strata means that some will be in higher or lower positions; others will have power, whereas others will be relatively powerless".

Max Weber (1864-1920) argued that social class was based on a person's market position which is basically how much money or wealth they have and their bargaining power to get this.

Karl Marx social theory acknowledged two social classes: Bourgeoisie who are the owners of the means of production and the Proletariat, the workers who have sold their labor, referred to as the exploited masses. According to Karl Marx, these are a people who are in a relationship to the means of production. The bourgeoisie own capital and the proletariat own their labor. Karl Marx views such relationships exploitative, shown by "surplus value" 
Karl Marx aimed to bring about a classless society where common means of ownership are practiced. He regarded capitalist society as exploitative as everything was determined by money and economics. In his view, ruling ideas are imposed on lower class, and this explains persistence of capitalism. In that regard, Karl Marx, argued, each social class should have its own ideology and system of beliefs. Karl Marx advocated for a revolution whereby society would be classless. This Marxist definition and interpretation of social class is paramount to the study. This is because society focused on access to healthcare based on socio-economic resources. The question as to who controls or directs the allocations of resources is of paramount importance to the study.

In the study, social class was operational zed on the basis of age, gender, income, education, occupation, marital status, wealth and place of residence. Socio-economic status in the study is broken into three levels: upper, middle and lower class. These are measured/operational zed social class based on the criteria shown below; age, gender, income, education, employment, marital status, and wealth. Socio-economic status is broken into three levels; upper, middle and lower, and assets are categorized as income, education and occupation. These are used to measure the social position of the household in the stratification of the society.

\subsection{Access to Public Healthcare-Dependent Variable}

Karl Marx viewed access to healthcare in terms of political power and economic dominance in a capitalist society. He argued that major improvements in health system could not occur without fundamental changes in broad social order. In his view, the health system mirrored the social order in society. He saw the bourgeoisie as the controllers of the health systems, health institutions, and the health workers were stratified according to the dictates of the capitalist system. This system could therefore not allow occupational mobility of the health workers.

Karl Marx argued that governments spend less on ill health, poor housing and institutional structures. In that view, ill health was viewed as a class problem that was related to social inequality. Access to healthcare was therefore class based, between the bourgeoisie and the proletariat (Bartley, 2004a, 2004b). The bourgeoisie who control most resources had better access to healthcare. On the other hand, the proletariat had less because of their social position in class. The solution to equitable access to healthcare was a transformation of socialism into communist state/society. This is a classless society, stateless human society based on common ownership; each according to his ability and needs. In this society, no one would have power over another and everyone should be equal. This ideal situation would give equal access to healthcare to everyone.

Access to healthcare is central in the performance of health systems around the world. Access to health is defined as a way of approaching, reaching a place, opportunity to reach a health facility. It has also been defined as access 
to a service, a provider, or an institution; access here is defined as the opportunity (Gulliford et al., 2002). Access to healthcare means helping people to command appropriate healthcare resources in order to preserve or improve their health. This implies that services should be available and adequate to supply the services required. People should have the opportunities to obtain healthcare, and in that way, they can access healthcare. Some definitions suggest that access means having timely use of personal health services to achieve the best health outcomes. In this context, coverage facilitates entry into the health system.

Access to healthcare has been conceptualized in several ways (Andersen \& Davidson, 2001). In this study access to healthcare, was measured/operationalized using the following dimensions: accessibility, approachability, acceptability, availability/accommodation, affordability and appropriateness. The model used shows the factors that lead to the use of health services. In this study, access to healthcare is determined by socio-cultural factors: need factors: culture, social resources, social capital, ethnicity, race, language spoken, discrimination, family support and poverty.

\section{Methods}

\section{Population}

Polit and Hungler refer to the population as an aggregate or totality of all the objects, subjects or members that conform to a set of specifications. In this study, the population, were all the households of all races/tribes, age groups, income levels, educational status, employment status, marital status, wealth status, residential areas who lived in Nairobi County during the survey. The people in the population must be in possession of specific characteristics in order to be included in the study. The eligibility criteria in this study were that the participants had to be residents in Nairobi County, have voluntarily accepted to participate in the study and that they also use health facilities in Nairobi County.

\section{Sampling techniques}

The procedure used to select a portion of the population to represent the entire population is sampling (LoBiondo-Wood \& Haber, 1998). A number of households who voluntarily accepted to participate in the study were selected. This procedure of selecting a sample to be studied rather than attempting to study the entire population of households saved money and time. This is so because obtaining data from the entire population, analyzing and interpreting would have been impossible to finish within the time constraints and limited financial resource.

\section{Sample}

A sample is a subset of a population selected to participate in the study; it is a fraction of the whole, selected to participate in the study. In this survey, a subset of 1066 households was selected out of the entire population of households who 
voluntarily accepted to participate in the study, in Nairobi County. Chadha's formula (2006) was used to determine the sample size:

Required information:-

- Anticipated population proportions $=P_{1} \& P_{2}$

- $\quad$ Confidence level $=95 \%$

- Absolute precision required on either side of the true value of the difference between proportions $=d$

Sample size can be estimated using the following formula:

$$
n=\frac{Z_{1-\frac{a}{2}}^{2}\left[P_{1}\left(1-P_{1}\right)+P_{2}\left(1-P_{2}\right)\right]}{d^{2}}
$$

$P_{1}, P_{2}=$ anticipated value of the proportions in the two populations.

For confidence level $95 \% z$ is taken as 1.96

Nairobi West

$$
P_{1}=\frac{212295}{985016}=0.2155=22 \%
$$

Nairobi East

$$
P_{2}=\frac{369866}{985016}=0.375=38 \%
$$

Nairobi North

$$
P_{3}=\frac{327428}{985016}=0.33=33 \%
$$

Westlands

$$
\begin{aligned}
& P_{4}=\frac{75427}{985016}=0.07=7 \% \\
& n=\frac{1.96^{2}\{0.22(1-0.22)+0.38(1-0.38)+0.33(1-0.33)+0.07(1-0.07)\}}{0.05^{2}} \\
& n=1066 \text { households }
\end{aligned}
$$

A total of 1066 households were surveyed using a random and multilevel sampling approach, respectively. These constituted the population for the study. As mentioned earlier, it was not always possible to cover all the population but this allowed for the highest accuracy to be obtained. The sample was therefore deemed reliable and appropriate to the study.

Multistage sampling is taking of samples in stages using smaller and smaller sampling units at each stage. This is a form of cluster sampling that involved several cycles of sampling. Counties were divided into clusters and then sampled. These selected clusters were further divided into smaller clusters and re-sampled again. This process was repeated till the ultimate sampling units were selected at the last of hierarchical levels.

Snowball technique was used to identify 10 households' members who dis- 
played the qualities or characteristics of interest to the study. After obtaining the information from the selected households, the researcher requested the respondents to assist in locating other respondents who would provide similar information. This method was found useful because the population with such characteristics were not easy to identify while others were unwilling to provide sensitive information.

A convenient sample comprising 20 health officials from the County was selected during the interview. A convenience sample is readily accessible persons in the study (De Vos 1998). These were health officials who were readily available and they fitted the criteria set for the study. However, the risk of bias is great, because each member of the population does not have an equal chance of being included in the sample. The results obtained may therefore not be generalized to the entire population.

$A$ stratified sample comprising 100 health workers was divided into different sub-groups according to their job titles, and then randomly selected for the study. Specialist group titles included medical officers, dentists, dental clinics, clinical officers, enrolled nurses, public health officials, pharmacists, technicians and others. This was found useful because each sub-group received proper representation within the sample. This also provides a better coverage of the population. The research has control over the subgroups to ensure all are represented.

This method was also used to select 20 health facilities for the study. The total population of health facilities was divided into sub-groups including national hospitals, county referral hospitals, district hospitals, sub-district hospitals, health centers, and dispensaries. Thereafter, they were randomly selected for the study. This enabled the researcher to high light each sub-group within the population. This method ensures the presence of each sub-group within the sample.

The study found it more economical to choose a sample of 1066 households, instead of studying the entire population of households in Nairobi County. The study also found it unnecessary to collect data from the entire population, as the responses from the sample were adequate to secure the information needed. This sampling technique was useful because of low costs and less time consuming; this suitable in situations where resources are limited.

\section{Data collection instruments}

In this study, data was collected using multiple techniques. The purpose of the study was to increase access to healthcare; to specifically determine the influence of selected factors in access to healthcare.

Qualitative data was collected using in-depth interviews and focus group discussions. The data collected was non-numerical. The participants were asked to respond to general questions and interviewers probed and explored the respondents to identify and define households' perceptions, opinions, and feelings about the phenomenon under study. 
In-depth interviews were conducted with key informants and samples of respondents. The interviewers invited between 6 - 10 participants to talk about the phenomenon. The interviewer made sure that the environment was pleasant and in some cases provided light refreshments to create a relaxed environment.

The objective of the in-depth interviews was to generate the following information: perceptions on services received, opinion on the benefits of health insurance, impact of the health facilities and attitudes on health facilities/ health workers.

Focus groups were classified according to the organization of the sessions. Some sessions were highly structured, whereas others were slightly structured. This was a focus group interview. Focus Group discussions explored the household's perceptions on factors that influence access to healthcare services. Here, the researcher designed specific topics that formed the subject of discussion. They were used for both male and females. In this study, FGDs were held in English and Kiswahili. However, in some parts of Embakasi and Kibera both languages were not understood. The research assistant had to engage an interpreter to help translate in the language understood. The information sought included: households' values, culture, opinions and attitudes about health facilities and health workers.

\section{Multivariate Analysis}

Qualitative data analysis was on socio-cultural variables in objective two. Socio-cultural factors analyzed included culture, language, social capital, poverty, race, ethnicity, migrant status, habits, values and others. The study hypnotizes that socio-cultural variables have an important influence on access to public care services.

Data were analyzed using various themes. These themes came from the review of the literature, characteristics of the phenomenon being studied, common sense, constructs, researcher's values, and personal experience with the subject matter. This is what ground theorists call open coding, and what content analysts call qualitative analysis or latent coding.

The transcripts were analyzed using thematic analysis and analytical phases included becoming familiar with the data, generating new codes, searching for and review for themes and patterns. The assistant researchers read each transcript a number of times in order to familiarize them with the content and thereafter generated initial codes. Items describing similar ideas were grouped, coded manually, and sorted to capture common themes. The team next reviewed, defined, named and identified the themes. The team conducted all analyses and resolved discrepancies through discussions. The objective here was to measure the relationship between socio-cultural factors and access to healthcare.

\section{Ethical Consideration}

The National Commission for Science, Technology and Innovation approved the 
study's research protocol on 14th July, 2015 for a period ending 18th December, 2015. Permit No. NACOSTI/P/15/7814/6977 was issued on 14th July. 2015. The Ministry of Health authorized the study to be carried out on its health facilities; The Ministry of education too authorized the study to be carried out; The County Government of Nairobi, Health Department authorized the study to be carried in the County.

Households in Nairobi County were eligible to participate in the study, 15+ years of age, and they live in Nairobi. Prior to participating, all individuals received a letter describing the study purpose and procedures, and that participation was voluntary. Oral and written consent was obtained from the participants before the interviews commenced. Al the data obtained was held confidential. The use of the data collected, the purpose and access to information as well as the role of the researcher was explained. The interviewers explained the usefulness of the study findings, which was to help plan improvements of access to public healthcare among various stakeholders.

\section{Findings}

This section uses qualitative data from observations, interviews, focus group discussions and in-depth interviews. The data collected shows the responses and perspectives of the respondents on attitudes towards new policies/regulations, culture, behaviors, attitudes, values, poverty, deprivations, social resources, social capital, communication, discrimination and so on. In this section, factors will not be referred to as variables. This is because in qualitative studies, we often do not use variables. However, there is a very thin line between independent and dependent variables. The study examined patterns and meanings of various factors above that affect access to healthcare.

\section{Attitudes towards new regulations}

Findings show that $10 \%$ of the respondents liked the new regulations on social insurance. They understood them and frequently benefitted from them. The $30 \%$ of the respondents somehow liked the new regulations. They partly used them to access healthcare. However, the majority $60 \%$ did not like the new regulations. They neither used them nor even understood them. According to them, these new regulations were a preserve of households of upper and middle class hierarchies. This failure to appreciate the regulations further alienated them for access healthcare.

The new regulations increased access among the upper and middle class social groups. On the other hand, the failure to appreciate new regulations undermined their capability to access healthcare. Attitudes therefore are important factors that affect access to healthcare. We therefore infer that attitudes can be interpreted to have significant influence on access to healthcare. Using content analysis, we use this behavioral data to classify, summarize and tabulate data. These are important factors that influence access to healthcare for all the groups, as shown in Table 1. 
Table 1. Attitudes towards new regulations.

\begin{tabular}{ccc}
\hline Social classes & Frequency & Percentages \% \\
\hline Upper class & 100 & 10 \\
Middle class & 302 & 30 \\
Lower class & 604 & 60 \\
\hline
\end{tabular}

Frequency distributions show the values of the observations and how often they occur. Responses from the lower class occur more frequently than both the upper and middle class. The percentages show the relationships between different sets. Attitudes and access to healthcare are mutually inclusive. There is a positive correlation coefficient between attitudes and access to healthcare.

\section{Perceptions and perspectives towards services provided}

The majority of the respondents, $60 \%$ lower class were not satisfied with the services provided by the County health systems. The healthcare delivery systems did not satisfy their needs as they are not treated well by the healthcare personnel. All kinds of treatments were quite unsatisfactory, and this limited their access to healthcare.

On the other hand, $30 \%$ of the middle class households expressed satisfaction with healthcare services. The services were not readily available but they were satisfied with what was available. This is so because this category had additional opportunities that gave them access to healthcare in private facilities. They had alternative options compared to the lower class.

But the $10 \%$ of the upper households had poor perceptions and perspectives but they did not care about services given. This category of social class had better socio-economic resources that gave them many options to access private healthcare. Whether services were available or not, was of less significance given the opportunities before them. Perceptions and perspectives were categorized and presented in frequencies and percentages as shown in Table 2.

Satisfaction with health services is important and tends to increase access healthcare. Consequently, satisfaction has a significant influence on access to healthcare. Satisfaction and access to healthcare were mutually inclusive as the association showed a positive relationship.

These relationships are expressed in frequencies and percentages. The frequencies show the value of the observations and how often they occur. They also help to compare the two data sets-satisfaction and access to healthcare. Percentages help to show the relationships between the data sets.

\section{Access to healthcare services}

$10 \%$ of the upper class were not very concerned about access to healthcare services. This is because they were not using public facilities much. Their healthcare was mainly in private health facilities, given that they had better opportunities to purchase private healthcare services. 
Table 2. Perceptions and perspectives.

\begin{tabular}{ccc}
\hline Social class & Frequency & Percentage\% \\
\hline Upper class & 100 & 10 \\
Middle class & 302 & 30 \\
Lower class & 604 & 60 \\
\hline
\end{tabular}

On the other hand, $30 \%$ of the middle class care somehow about access to healthcare services. They partly used public facilities and therefore accessibility was to some extend important. This was because they had some socio-economic packages that gave them some options to use private healthcare.

However, the majority $60 \%$ of the lower class viewed access to healthcare as a very important component of their healthcare. They entirely depend on public health services and any delays or otherwise seriously affected them. This is so because they entirely depend on public facilities to access healthcare. They have no opportunities, given their deprived status to access healthcare. These perceptions on access to healthcare as shown in Table 3.

Access to healthcare was a very important factor and it determined healthcare among the people. Access to healthcare was an important factor that determined the nature of access people achieved. Positive attitudes towards access to healthcare are important. Positive attitudes are mutually inclusive to access to healthcare.

The data is displayed infrequency tables in order to show the number of times each score occurs. The percentages are used to compare the information displayed. The results show a positive association between the factors. The correlation shows a strong statistical relationship.

\section{Communication}

The findings show that $50 \%$ of the lower class did not understand English or Kiswahili. They could only communicate in their local languages. The health facilities lacked interpreters to help them communicate with health providers. This affects their ability to access healthcare.

I addition, 30\% of middle class had fairly good communication skills and could communicate with health providers well. They could communicate in English and Kiswahili. This medium communication increased their access to healthcare. This advantage was due to their advantaged socio-economic status.

Further, $20 \%$ of the upper class had very good communication with the health providers. They communicate easily with health providers, and this increases their access to healthcare. This is partly due to their socio-economic status.

Consequently, ability to communicate was an important factor in access to healthcare. It influenced access to healthcare, as shown in Table 4.

Communication was mutually inclusive to access to healthcare. Communication has a positive relationship with access to healthcare. The correlation was statistically significant, mutually inclusive and highly correlated. Frequency and percentages show the occurrence of the data sets and their relationships respectfully. 
Table 3. Access to health services.

\begin{tabular}{ccc}
\hline Social class & Frequency & Percentages\% \\
\hline Upper class & 100 & 10 \\
Middle class & 302 & 30 \\
Lower class & 604 & 60 \\
\hline
\end{tabular}

Table 4. Communication factors.

\begin{tabular}{ccc}
\hline Social class & Frequency & Percentages \\
\hline Upper class & 201 & 20 \\
Middle class & 302 & 30 \\
Lower class & 503 & 50 \\
\hline
\end{tabular}

\section{Lack of faith in Public Facilities}

The findings show that $10 \%$ of they had no faith in health facilities. These facilities were dilapidated, old and needed repairs urgently. They lacked efficient health workers. In addition health facilities lacked clean water and sanitation. They shied away from using them in preference to private health facilities.

On the other hand, $30 \%$ of middle class had some limited faith in public health facilities. They used them partly to supplement their use in private facilities. This was so because they had limited access to socio-economic opportunities compared to the upper class.

However, $60 \%$ had faith in public facilities despite lack of essential facilities like laboratories, clean water and sanitation and few health personnel. Even without faith, lower class entirely relies on public facilities for healthcare. They lack socio-economic resources and cannot afford private healthcare, as shown in Table 5.

Faith was therefore an important factor that had a significant influence on access to healthcare. Faith and access to healthcare are mutually inclusive and highly correlated. Pearson correlation is significant at the 0.01 level (2-tailed). The correlation shows the statistical relationship between faith and access to healthcare,

\section{Cultural factors}

The study shows that $50 \%$ of lower social class respect culture/ because it affects perceptions of health, illness and death. They value customs, values, language and traditions as they make it easy to communicate freely and honestly. In this way, they can reduce disparities and improve health outcomes.

On the other, $40 \%$ have some respect for cultural components because their socio-economic positions have added considerable challenges to culture and traditions. They consider culture and traditions as somehow outdated and they prefer modern western culture. Cultural factors therefore have limited effect on access to healthcare. 
Table 5. Faith in health facilities.

\begin{tabular}{ccc}
\hline Social class & Frequency & Percentage \\
\hline Upper class & 101 & 10 \\
Middle class & 503 & 50 \\
Lower class & 402 & 40 \\
\hline
\end{tabular}

However, $10 \%$ of the upper households have limited respect to culture and related traditions. They consider culture outdated and outrageous and therefore try their best to discard in preference to modern culture. Most of these households have high opportunities occasioned by their access to socio-economic assets-high income, education and occupations. These factors have profoundly changed their lifestyles and now only prefer to use modern medicine. They shun culture because it makes them look backward and primitive, as shown in Table 6 .

Cultural factors have a vast influence on health, especially among the lower social class. They affect their perceptions on various dimensions on health and dieses. They influence where they go when they are ill, types of healthcare and in fact types of treatment they seek. Correlation coefficient is strong between cultural dimensions and access to healthcare, especially among the lower class as shown in the frequencies and percentages above. Pearson correlation shows that the two factors are mutually inclusive and highly correlated at 0.01 level (2-tailed).

\section{Attitudes towards health workers}

In the study, $50 \%$ of the lower class experienced hostile receptions; the workers were rude and lacked cultural appreciation. These in depth results show that these households were of lower socioeconomic status. Despite these negative attitudes, they still seek healthcare in public places, due to limited options arising from their socio-economic deprivations. These attitudes seriously affect access to healthcare.

On the other hand, $30 \%$ of middle class had some negative attitudes towards health workers. This forced them to seek healthcare in private facilities, where staff are respectable. They are able to do so because they have better access to socio-economic resources. These opportunities give them alternative options.

However, $20 \%$ of the upper class had very negative attitudes but cared less because they had alternatives.

They were of high socio-economic status-high incomes, education, and employment and therefore they do not rely so much on public health facilities for their health needs. They have some sympathy with the workers and do not have such adverse observations, as shown in Table 7.

Attitudes to healthcare workers have an influence on access to healthcare. Pearson Product Moment correlation shows that there is association between the two factors; attitudes and access to healthcare, as shown by the ratios and interval scales on the table above. These factors are mutually inclusive and highly correlated. 
Table 6. Cultural factors.

\begin{tabular}{ccc}
\hline Social class & Frequency & Percentage \% \\
\hline Upper class & 101 & 10 \\
Middle class & 402 & 40 \\
Lower class & 503 & 50 \\
Total & 1006 & 100 \\
\hline
\end{tabular}

Table 7. Attitudes towards health workers.

\begin{tabular}{ccc}
\hline Social class & Frequency & Percentages \\
\hline Upper class & 201 & 20 \\
Middle class & 302 & 30 \\
Lower class & 503 & 50 \\
Total & 1006 & 100 \\
\hline
\end{tabular}

\section{Social resources}

The results show that $20 \%$ of upper social class respect social resources. Social resources include tangible items like money, information, goods and services. Love and affection fall in this category. Social position in society is part of social resources. Social resources do not influence because they are socially high in the social hierarchy and have many social goods like high incomes, education and employment that gives them a privileged position in society. They can access healthcare in private facilities and cannot therefore depend on traditions to influence their access.

On the other hand, $30 \%$ of middle class have fair respect or value to social resources. They too have some fairly moderate social goods, their social economic status in society. They have fairly good incomes, education and employment opportunities. These factors influence their access to healthcare.

However, the majority $50 \%$ value social resources as they have considerable influence on access to healthcare. These factors frame behavioral choices including decisions affecting health. Many of these households find themselves engage in high risk behaviors that include drinking, smoking and unhealthy diets. All these affect access to healthcare. They engage in these activities because they lack socio-economic goods that can make them change their behavior. They engage in these activities because they have limited opportunities.

Pearson correlation shows that there is a significant relationship between the two factors, social capital and access to public healthcare. This correlation is significant at 0.01 level (2-tailed). The results confirm that the two factors are significantly correlated: thus mutually inclusive and highly correlated.

\section{Social capital}

The results show that upper class $10 \%$ of upper class value social capital and the rest have no respect or value to it. Social capital includes dimensions like economic resources gained from being part of net works of social relationships, 
trust, trustworthiness, and civil norms, association of membership, voluntary associations, and homogeneity. Social net works, social support, social networks and social connections are valuable to households and allow access to healthcare resources (Aye et al., 2002). They sometimes provide job opportunities and help enhance skills.

The majority do not value social capital because they are insulated by high socio-economic resources. They have high incomes, education and employment opportunities and these resources promote their health needs.

On the other perspective, $20 \%$ of the middle class had some middle level value for social capital. This was because they sometimes benefit from social capital. This position they hold is because they also have some social goods like moderate incomes, education and employment opportunities that enable them access healthcare resources but in some limited way.

However, the majority $70 \%$ of the lower class have considerable value for social capital. High level of social capital does influence access to healthcare through spread of health norms. Social capital increase knowledge and skills that affect access to healthcare. It also helps tackle health inequalities that result from social isolation, low levels of support and confidence. Social capital sometimes tries to reduve the gap between the poor and the rich. Furthermore, it helps to increase support opportunities for people and groups to form connections. Negative social capital can also pose restrictions of individual freedom and exclusion, as shown in Table 8.

The results show that social capital has influence on access to healthcare. The two factors as shown by Pearson Product Moment Correlation confirm that there is association between the two factors, measured at ratio or interval scales. The two factors are mutually inclusive and highly correlated. This relationship is significant at 0.01 level (2-tailed).

\section{Discussion}

Socio-cultural factors have also been found to have a significant influence on access to public healthcare. These factors include culture, attitudes, values, discrimination, stress, associations, and social capital among others. Stress for example has been found to affect access to healthcare in many instances: Stress has been associated to risk behavior and chronic diseases and these are dominant causes of mortal. Quite often, people do not take stress as important for their health let alone the fact that some do not even understand when stressed. This continues to be a killer disease albeit lack of specific policies to address the vice.

Other socio-cultural factors include lack of "social resources" among the lower socio-economic classes. These include character and intensity of social net works, associational social hierarchies (Hall et al., 2009). These resources are important and they assist households access resources that are adequate to meet their healthcare needs. It is important that these resources are nurtured and distributed evenly among the households. 
Table 8. Social capital.

\begin{tabular}{ccc}
\hline Social Class & Frequency & Percentage \% \\
\hline Upper class & 101 & 10 \\
Middle class & 201 & 20 \\
Lower class & 704 & 70 \\
Total & 1006 & 100 \\
\hline
\end{tabular}

Other factors related to the above are social relations, social net works, trust, secondary associations, marriages and friendships. All these constitute "social capital" that is critical for day-to-day living. Others include status hierarchies in society that create distinctions among social class, and assign individuals with prestige social positions. These factors (independent variables) have profound influence on access to public healthcare (dependent variable). Lack of them increases inequality in access to healthcare.

Unfortunately, little is being done to augment these factors and these have increased stress leading to premature deaths. Some studies have shown that such people appear to be healthier (Kawachi et al., 2004). Such illusions have resulted into fatal health outcomes. It is imperative that government and other stokeholds address the need to have these factors so that they can enhance the health. Relevant departments of government should increase these among the people who need them most.

\section{Conclusion}

This study is on "Social class and access to Public Healthcare in Kenya, A case For Nairobi County". This research is important because it provides important information about access to healthcare and related outcomes and interventions. Access to healthcare faces many barriers and this study attempted to address their influences and suggest possible interventions. Access to healthcare is important because it prevents diseases, reduces them and prevents early deaths. It helps also increase life expectancy among the population. However, these gains are threatened by factors that affect access to healthcare. These factors include poverty, geographical area of residence, race and ethnicity, sex, age, language spoken, and disabilities. They affect ability to access healthcare, availability, timeliness, convenience and affordability.

Access to healthcare in the County is unequal among the households. Some households of upper social hierarchy, access healthcare more than say the middle and lower social classes. The lower social class end with worse health outcomes compared to the middle or upper social hierarchies. This health inequality has some correlation with income inequality. The higher social economic social classes have better access to socio-economic resources and opportunities compared to the lower social class. These socio-economic opportunities include high incomes, education, occupation and wealth; and are unevenly distributed in 
favor of the upper and middle class social hierarchies.

These social goods give better advantages to the upper and lower social classes. They access more healthcares. The higher the level of incomes, education, occupation and wealth is, the more the opportunities for accessing to healthcare are. Unequal access to healthcare contributes to disparities in health status. It increases costs for both the insured and the uninsured. This also means that there are less disposable incomes, particularly burdening the lower social income households. Categories of households in this category cannot afford the care they need and this has forced them to forego such care all together.

The findings show that health inequalities are further exacerbated by unequal distribution of incomes, power and wealth. Lack of these factors increase poverty and marginalization among the lower social classes. These factors further affect socio-cultural factors. Absence of social capital, social resources like associations and marriages, unemployment and job loses increase deprivations and, hence affect access to healthcare.

\section{Acknowledgements}

This work could not have been successful without the contribution of various institutions, institutions and organizations. I appreciate the useful information, materials and support I received from the field study from Office of the President, the Ministry of Health, Ministry of Planning and Devolution, Ministry of Education and the County Government of Nairobi County. Special thanks go to the Office of the President for partial scholarship and study leave during the time I was conducting the research. I cannot forget to thank the University of Nairobi, Department of Political Science and Public Administration for their enduring support during the study. In equal measure, I thank all the staff for facilitating my field study.

\section{Conflicts of Interest}

The authors declare no conflicts of interest regarding the publication of this paper.

\section{References}

Andersen, R. M., \& Davidson, P. L. (2001). Improving Access to Care in America: Individual and Contextual Indicators. In R. M. Andersen, T. H. Rice, \& E. F. Kominski (Eds.), Changing the U.S. Health Care System: Key Issues in Health Services, Policy, and Management. San Francisco, CA: Jossey-Bass.

Avila, R. M., \& Bramlett, M. D. (2013). Language and Immigrant Status Effects on Disparities in Hispanic Children's Health Status and Access to Health Care. Maternal and Child Health Journal, 17, 415-423. https://doi.org/10.1007/s10995-012-0988-9

Aye, M., Champagne, F., \& Contandriopoulos, A. P. (2002). Economic Role of Solidarity and Social Capital in Accessing Modern Health Care Services in the Ivory Coast. Social Science \& Medicine, 55, 1929-1946. https://doi.org/10.1016/S0277-9536(01)00322-7

Barr, D. A. (2008). Health Disparities in the United States: Social Class, Race, Ethnicity, 
and Health. Baltimore, MD: John Hopkins University Press.

Bartley, M. (2004a). Health Inequalities and Social Institutions. Social Theory \& Health, 68, 63-79.

Bartley, M. (2004b). Health Inequality: An Introduction to Theories, Concepts and Methods. Cambridge: Polity Press.

Brawley, M. (2000). The Client Perspective: What Is Quality Health Care Service. A Literature Review. In Delivery of Improved Services for Health: USAID Cooperative Agreement (pp. 617-700).

Brotherton, S. E. et al. (2005). US Graduate Medical Education 2004-2005: Trends in Primary Care Specialties. JAMA, 294, 1075-1082. https://doi.org/10.1001/jama.294.9.1075

Chadha, V. K. (2006). Sample Size Determination in Health Studies. NTI Bulletin, 42, 55-62.

Donabedian, A. A. (1990). The Seven Pillars of Quality. Archives of Pathology \& Laboratory Medicine, 114, 1115-1118.

Durham, J. et al. (1998). Self-Assessed Health Status and Selected Behavioral Risk Factors among Persons with and without Health Coverage United States-1994-1995. MMWR, 47, 176-180.

Gulliford, M., Figueroa-Munoz, J., Morgan, M., Hughes, D., Gibson, B., Beech, R., \& Hudson, M. (2002). What Does “Access to Health Care" Mean? Journal of Health Services Research \& Policy, 7, 186-188. https://doi.org/10.1258/135581902760082517

Hall, P. et al. (2009). Successful Societies: How Institutions and Culture Affect Health. Cambridge: Cambridge University Press. https://doi.org/10.1017/CBO9780511816192

Kawachi, I. et al. (2004). A Glossary for Health Inequalities. Journal of Epidemiology \& Community Health, 56, 647-652. https://doi.org/10.1136/jech.56.9.647

Schellenberg, J. et al. (2003). Tanzania IMCI MCE Baseline Household Survey Study Group. Inequities among the Very Poor: Health Care for Children in Rural Southern Tanzania. The Lancet, 361, 561-566. https://doi.org/10.1016/S0140-6736(03)12515-9

Starfield, B. et al. (2004). The Medical Home, Access to Care and Insurance. Pediatrics, 113, 1493-1498.

Wilkinson, R. G. (1976). The Epidemiological Transition: From Material Scarcity to Social Disadvantage? Daedalus, 123, 61-77. 